\title{
Angle-independent microwave absorption by ultrathin microcavity arrays
}

\author{
J. R. Brown, ${ }^{1, a)}$ A. P. Hibbins, ${ }^{2}$ M. J. Lockyear, ${ }^{2}$ C. R. Lawrence ${ }^{3}$ and J. R. Sambles ${ }^{2}$ \\ ${ }^{1}$ Omni-ID, The Enterprise Centre, Farnham GU10 5EH, United Kingdom \\ ${ }^{2}$ School of Physics, University of Exeter, Devon EX4 4QL, United Kingdom \\ ${ }^{3}$ QinetiQ, Cody Technology Park, Farnborough GU14 OLX, United Kingdom
}

(Received 26 April 2008; accepted 19 June 2008; published online 28 August 2008)

\begin{abstract}
The resonant absorption of microwave radiation by thin, two-dimensional microcavity arrays has been studied. Resonant modes associated with these structures, formed from copper-clad FR4 laminates, exhibit both an azimuthal and polar angle independent electromagnetic response. The experimental data agree well with the predictions of a finite element method computer model, which has been utilized to explore the electromagnetic character of the resonant modes supported. () 2008 American Institute of Physics. [DOI: 10.1063/1.2970081]
\end{abstract}

\section{INTRODUCTION}

Conventional materials for the attenuation of millimetric electromagnetic radiation have historically been limited to a minimum thickness of a quarter-wavelength, with examples including Salisbury screens ${ }^{1,2}$ and Dallenbach layers. ${ }^{1}$ This limitation can present significant practical problems in terms of thickness and weight penalties incurred. Hibbins et al. ${ }^{3}$ used ultrathin cavities as resonant absorbers, which are two metal layers spaced by a dielectric whose thickness is grossly subwavelength, the uppermost of which is perforated periodically by a single set of continuous subwavelength slits. This type of monograting geometry has also been applied to produce absorption in the optical regime. ${ }^{4}$

Others ${ }^{5,6}$ used two orthogonal sets of subwavelength slits and thus divided the upper metal layer into an array of square patches. These bigrating structures are capable of guiding electromagnetic waves and therefore have myriad applications in communications and electronics. Zhang et al. ${ }^{5}$ analyzed such a square patch array as a perfect magnetic conductor $^{7}$ and, using a transmission line model, applied the structure to suppress side lobes from an antenna array. A previous work by Sievenpiper et al. ${ }^{6}$ involved the introduction of vias and varactor diodes to a square grating to create an electrically tuned device for use in beam steering and can also be used as an absorber. ${ }^{8}$ Other approaches include those of Tennant and Chambers, ${ }^{9,10}$ who created adaptive radar absorbers using arrays of dipole elements and $p-i-n$ diodes. In this study we show that similar ultrathin bigrating (twodimensional) metal-dielectric-metal structures exhibit strong absorption bands that are almost completely independent of the angle of incidence and sample orientation. The resonant modes produced by the structure are identified, and their variation in response with azimuthal (rotation) and polar incident angles is investigated. Experimental data are compared to the predictions of a finite element model, ${ }^{11}$ thus allowing the nature of the resonant modes to be examined.

\section{EXPERIMENTAL DETAILS}

The experimental coordinate system and sample geometries are shown in Fig. 1. The samples are formed from

${ }^{a)}$ Electronic mail: j.r.brown@exeter.ac.uk.
$356 \mu \mathrm{m}$ thick FR4 printed circuit board made from laminated glass cloth infused with resin and bounded at the top and bottom by $18 \mu \mathrm{m}$ thick copper layers. The period of each sample is $10 \mathrm{~mm}$, each slit having a width of $0.3 \mathrm{~mm}$, formed using standard print and etch techniques. ${ }^{12}$ Their specular reflectivity is measured as a function of azimuthal and polar angles using a system comprising a scalar network analyzer connected to reference and signal detectors and a pair of horn antennas. The sample is placed on a turntable, the rotation of which is controlled by a stepper motor. Collimated radiation from the transmit antenna is directed onto the sample using a spherical mirror mounted above it. The polarization state of the antennas can be varied individually, allowing for the response to $p$-(TM) and $s$-(TE) polarized radiation to be recorded. Measurements are taken at polar angles $(\theta)$ between $16^{\circ}$ and $73^{\circ}$ and the sample rotated between $0^{\circ}$ and $90^{\circ}$ azimuths $(\phi)$, with the full azimuthal behavior being obtained from the rotational symmetry of the sample.

\section{RESULTS}

The response of the monograting under azimuthal rotation has been studied to establish a benchmark against which the response of the bigrating can be compared. While results from a similar geometry have previously been reported, ${ }^{3}$ its electromagnetic response as a function of azimuthal angle (i.e., rotation) was not considered. As demonstrated then, the sample supports a TEM waveguide mode ${ }^{13}$ within the dielectric layer. This mode resonates in a region beneath the metallic regions of the illuminated surface in a similar manner to the Fabry-Pérot-like resonances of a metallic slit. ${ }^{14}$ Consider the rotation of the sample away from $\phi=0^{\circ}$ and resolution of the incident momentum into the $x$ - and $y$-directions. Assume infinitesimal slit width and set $\lambda_{y}=2 \lambda_{g}$ for the fundamental standing wave resonance. ${ }^{3}$ It can then be shown using the equation

$$
n^{2} k_{0}^{2}=k_{x}^{2}+k_{y}^{2},
$$

where $n$ is the refractive index of the incident medium $(n$ $=1$ for vacuum), $k_{0}$ is the wavevector of the free space wave, $k_{x}$ is the wavevector of the wave in the core in the 


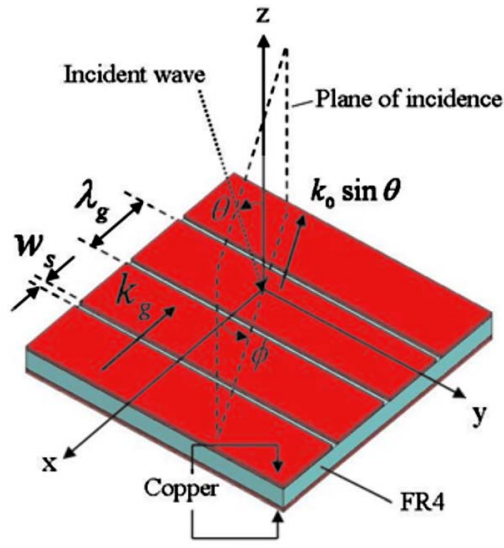

(a)

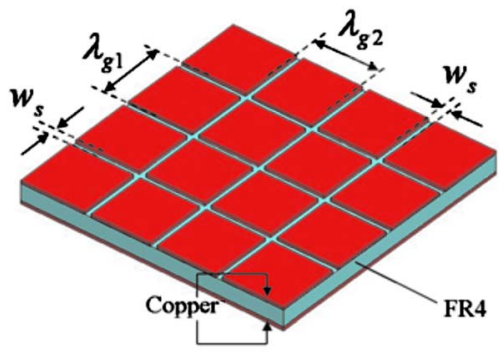

(b)
FIG. 1. (Color online) (a) The monograting sample geometry (not to scale) and the coordinate system used: $\theta$ is the polar angle, $\phi$ is the azimuthal angle, $\lambda_{g}$ $=10 \mathrm{~mm}$, and $w_{s}=0.3 \mathrm{~mm}$. (b) Three-dimensional projection of the bigrating $\lambda_{g 1}=\lambda_{g 2}$. (c) Cross section through the bigrating structure, $t_{m}=18 \mu \mathrm{m}, t_{c}$ $=356 \mu \mathrm{m}, w_{s}=0.3 \mathrm{~mm}, \lambda_{g 2}=\lambda_{g 1}=10 \mathrm{~mm}$, and sample area of $500 \times 500 \mathrm{~mm}^{2}$.

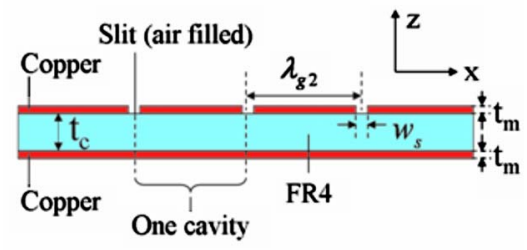

(c)

$x$-direction, and $k_{y}$ is the wavevector of the wave in the core in $y$-direction, that

$$
\lambda_{0}^{2}=\left(2 n \lambda_{g}\right)^{2}\left(1-\sin ^{2} \theta \sin ^{2} \phi\right),
$$

where $\lambda_{0}$ is the free-space wavelength.

The grayscale plots of Figs. 2(a) and 2(b) show the respective specular $R_{p p}$ and $R_{s s}$ reflected intensities from the sample. The subscripts refer to the incident and detected polarizations, respectively. The data are plotted as a function of frequency and azimuthal angle with $\theta=16^{\circ}$.

The dark bands at 7 and $14.6 \mathrm{GHz}$ in Fig. 2(a) indicate strong absorption. Using Eq. (1) from Ref. 3 for the standing wave condition with $\varepsilon_{r}=4.17-0.07 i$ for the relative permittivity of the dielectric core, ${ }^{3}$ one can identify the mode at $\approx 7 \mathrm{GHz}$ as the fundamental resonance. Similarly, the mode

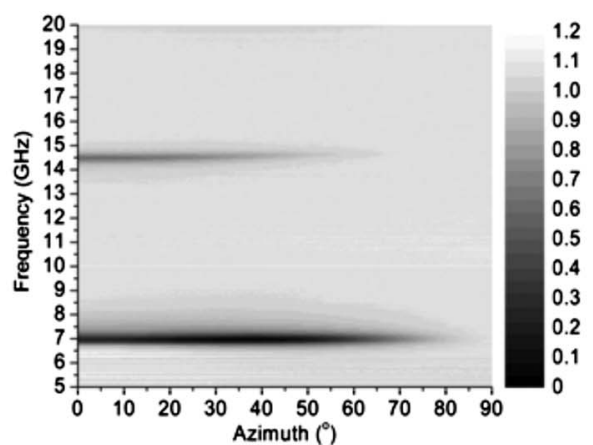

(a)

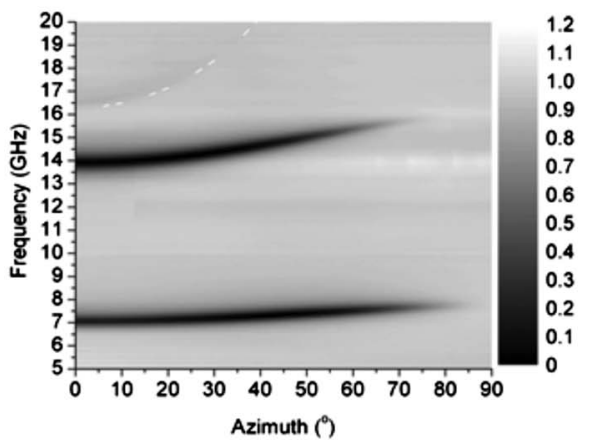

(c)

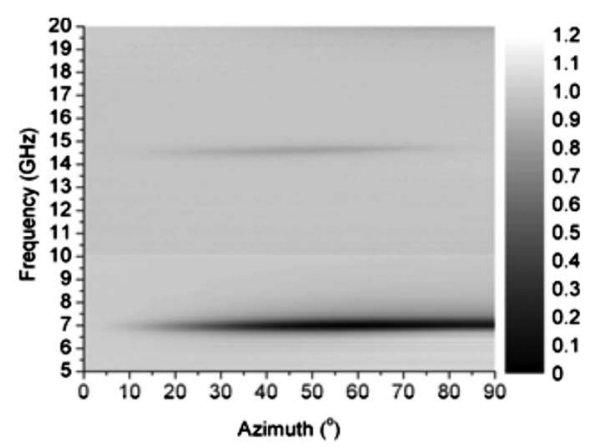

(b)

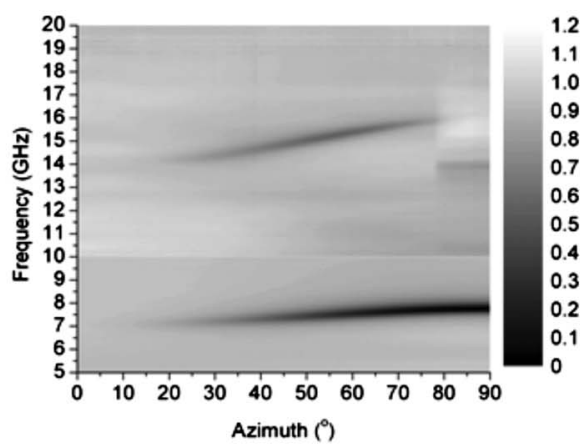

(d)
FIG. 2. Monograting sample. (a) Experimental $R_{p p}$ data as a function of frequency and azimuthal angle at $\theta$ $=16^{\circ}$. (b) Experimental $R_{s s}$ data as a function of frequency and azimuthal angle at $\theta=16^{\circ}$. (c) Experimental $R_{p p}$ data as a function of frequency and azimuthal angle at $\theta=57^{\circ}$. (d) Experimental $R_{s s}$ data as a function of frequency and azimuthal angle at $\theta=57^{\circ}$. 
at $\approx 14.6 \mathrm{GHz}$ is the second harmonic. The fundamental resonance is strongest at $\phi=0^{\circ}$ where the component of the incident electric field vector perpendicular to the slits is greatest. It becomes progressively shallower as the grating is rotated, with no resonance occurring at $\phi= \pm 90^{\circ}$ since there is no component of the electric field across the slit. Conversely, the fundamental absorption band in Fig. 2(b) for $s$-polarized radiation shows greatest absorption at $\phi= \pm 90^{\circ}$ and no absorption at $0^{\circ}$. Again this is due to the electric field lying parallel to the slits for $\phi=0^{\circ}$ with $s$-polarization. Figures 2(c) and 2(d) are $R_{p p}$ and $R_{s s}$, respectively, for the monograting at $\phi=57^{\circ}$. Now the bands demonstrate a much higher degree of curvature in order to satisfy the conservation of momentum [Eq. (2)]. With reference to Fig. 2(c), the faint, highly curved band centered on $\phi=0^{\circ}$ is due to conical diffraction. The superposition of the incident momentum with that supplied by the grating results in a quadratic equation in $k_{0}$,

$$
k_{0}^{2}\left(1-\sin ^{2} \theta\right)-k_{0}\left(2 k_{g} \sin \theta \cos \phi\right)-k_{g}^{2}=0 .
$$

The solution of Eq. (3) yields the limit frequency at which diffraction will occur, and this solution has been plotted as a dashed line in Fig. 2(c).

One important distinction between the responses of the monograting to $p$-polarized and $s$-polarization radiations concerns the frequency stability of the fundamental mode with changing polar angle. At an azimuthal angle of $0^{\circ}$ the frequency given by Eq. (2) is independent of the polar angle; hence, the resonance is invariant. At this angle, coupling to the mode from $p$-polarized radiation is maximized. There can, however, be no coupling from $s$-polarized radiation. By comparison, the $s$-polarized mode is optimally coupled at an azimuthal angle of $90^{\circ}$ at which angle the frequency given by Eq. (2) is strongly dependent on the polar angle. The primary consequence of this is a difference in the frequency dependence of the $p$ - and $s$-polarized absorption bands at their respective optimally coupled azimuthal angles.

The second order mode for $s$-polarized radiation, shown as the dark bands between 14 and $17 \mathrm{GHz}$ in Figs. 2(b) and 2(d), cannot be coupled at $\phi= \pm 90^{\circ}$ (unlike the fundamental mode). This can be understood by considering the behavior of the standing wave fields within the core. The component of the incident electric field that lies perpendicular to the slits creates regions of enhanced charge density of opposite sign on either side of the slits. ${ }^{3}$ Between the slits the fundamental mode undergoes a phase change of $\pi$-radians, as expected for a simple organ-pipe-type resonance. The second order mode, however, undergoes a phase change of $2 \pi$ rad between the slits. When added to the enforced $\pi$-radian phase change across the slit, this results in a total phase change of $3 \pi \mathrm{rad}$ within one period of the structure. Therefore, at any given slit, the field resulting from the wave coupled in through that slit will be $\pi$-radians out of phase with the field coupled in through the adjacent slits. This results in destructive interference and prevents propagation of the mode. To permit propagation of the second order mode, some additional phase offset is needed. At normal incidence the fields are in phase at all slits regardless of the orientation of the electric vector, and hence the second order mode can never

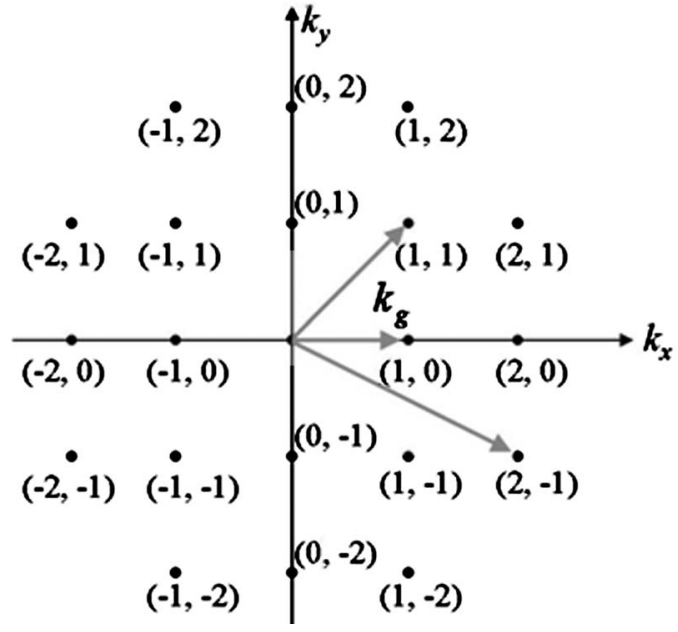

FIG. 3. Reciprocal space diagram of the bigrating.

be excited. Off normal there is a phase difference between slits for $p$-polarized radiation at all azimuths, but for $s$-polarization, there is no phase difference between slits at $\phi= \pm 90^{\circ}$ regardless of the polar angle, and hence no mode is excited at these orientations.

\section{A. Bigratings}

In order to fully understand the modes supported by the bigrating sample, it is useful to represent its periodicity on a reciprocal space diagram. Two arrays of slits of identical spacing, etched orthogonally to one another, yield a twodimensional array of lattice points [Fig. 3]. The lattice points can be grouped into sets; for example, the set $\{1,1\}$ includes the $(1,1),(-1,1),(1,-1)$, and,$(-1,-1)$ individual points. Note the different parentheses used to represent a set of points versus an individual point. Therefore, in addition to the $\{0,1\}$ set of lattice points associated with each monograting, a set of $\{1,1\}$ points also exists. This makes it possible to couple to a set of modes that are inaccessible for the monograting.

A grayscale plot showing $R_{p p}$ at $\phi=57^{\circ}$ for the bigrating is presented in Fig. 4(a). There are four dark bands indicating absorption at $\approx 7, \approx 11, \approx 14.5$, and $\approx 17 \mathrm{GHz}$. A cross section through the grayscale plot for a fixed azimuthal angle of $45^{\circ}$ is shown as a line plot in Fig. 4(c). Also shown is the reflectivity as predicted by a finite element model $^{11}$ for the same incident angles. Excellent agreement between the measured data and the finite element prediction is obtained using the previously determined ${ }^{3}$ values of permittivity for FR4 $\left(\varepsilon_{r}=4.17+0.07 i\right)$ with a period of $10 \mathrm{~mm}$ and a slit width of $0.3 \mathrm{~mm}$.

Returning to Fig. 4(a), the addition of the second set of slits enables coupling to the fundamental $\{1,0\}$ set of modes at $7 \mathrm{GHz}$ at all azimuthal angles, as there is always a component of the electric field perpendicular to at least one set of slits. At $\phi=90^{\circ}$ the coupling is to the $(1,0)$ mode, whereas at $\phi=0^{\circ}$ the coupling is to the $(0,1)$ mode; for $0^{\circ}<\phi<90^{\circ}$ both modes are excited. Strikingly, unlike in the monograting case, there is no significant curvature of the band. This is due to the interaction of these two modes. 


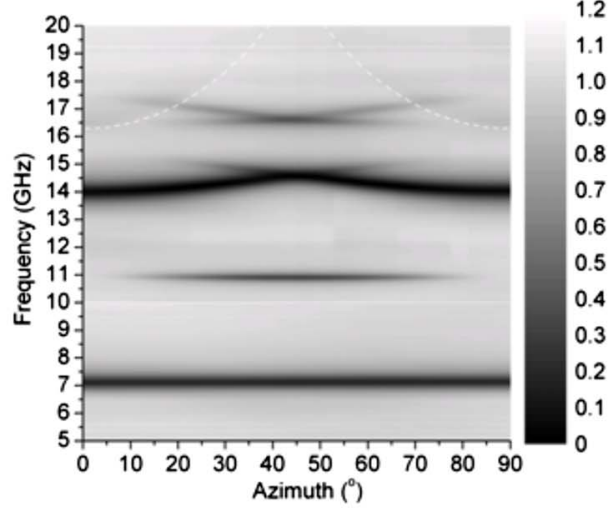

(a)

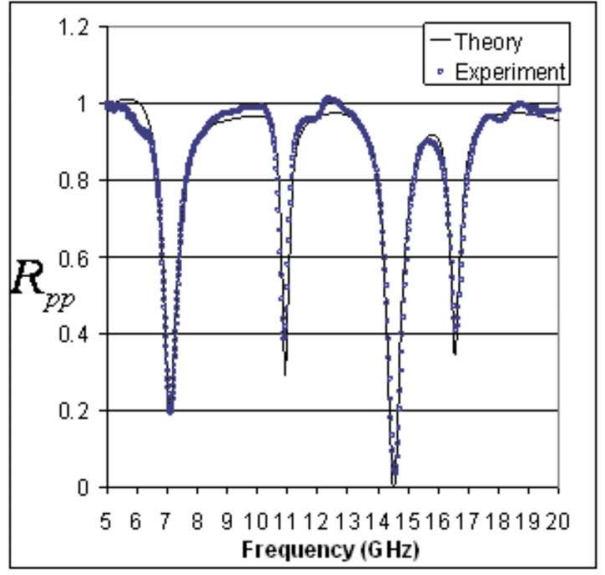

(c)

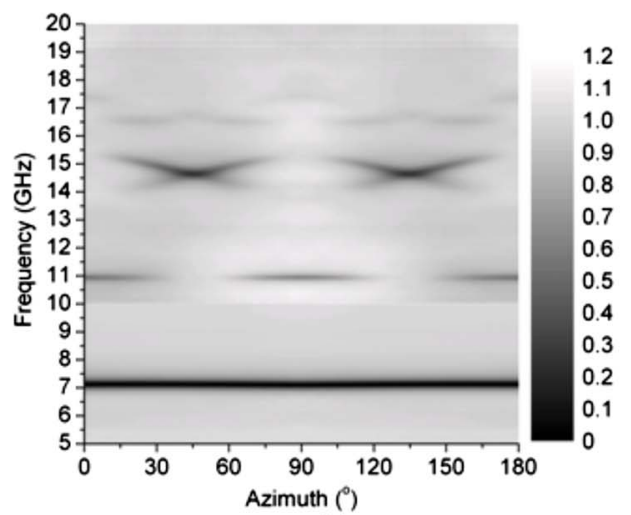

(b)

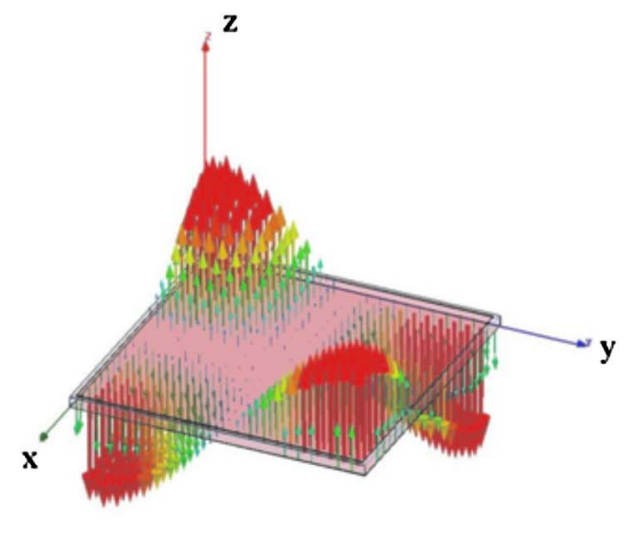

FIG. 4. (Color online) Bigrating sample. (a) Experimental $R_{p p}$ data as a function of frequency and azimuthal angle at $\theta=57^{\circ}$. (b) Experimental $R_{s s}$ data as a function of frequency and azimuthal angle at $\theta=57^{\circ}$. (c) Line plot showing comparison of measured data to the predictions of the numerical model: $R_{p p} \theta=57^{\circ}, \phi=45^{\circ}$. (d) Prediction of the electric field vector distribution at a phase corresponding to peak field strength on the upper surface of the lower metal layer for a $\{1,1\}$ mode at $10.93 \mathrm{GHz}$. The longest arrows correspond to enhancements of 13 times the injected field.
The mode observed at $\approx 11 \mathrm{GHz}$ in Fig. 4(a) is due to the $(1,1)$ scattering and has no equivalent on the monograting sample. Considering Eq. (1), $k_{x}$ and $k_{y}$ must be equal, giving

$$
n k_{o}^{2}=2 k_{x}^{2},
$$

and hence the frequency of the $\{1,1\}$ modes should be $\sqrt{2}$ times greater than that of the $\{1,0\}$ modes. Inspecting Fig. 4(a) and taking the ratio of the resonant frequencies return a value of $\approx 1.5$. The $(1,1)$ mode suffers no curvature because rather than a superposition of two orthogonal modes, it is a single mode of oscillation that can be coupled to from either the $x$ - or $y$-direction or at any angle in between. The total momentum available to this mode therefore does not change with the azimuthal angle; hence, the mode is flat banded. Also note that the $\{1,1\}$ modes cannot be coupled to for $\phi$ $=0^{\circ}, \pm 90^{\circ}$. We explain this character by using the finite element model ${ }^{11}$ to examine the mode's field distribution.

Figure 4(d) is a plot of the electric field vector at a phase corresponding to the maximum field on the upper surface of the lower metal layer for a $\{1,1\}$ mode: the strongest electric field and therefore the greatest charge densities occur at the corners with diagonally opposite corners having charge accumulations of the same sign. In order to couple to a $\{1,1\}$ mode, this charge distribution must be created by the electric field of the incident wave. For a $p$-polarized wave incident at $\phi=0^{\circ}$, this charge distribution cannot be set up at any polar angle. The incident electric field is parallel to the $y$-axis and results in the accumulation of a net positive charge along one side of the patch and a net negative charge on the opposite side [see Fig. 5(a)].

An $s$-polarized wave incident at the same azimuth $(\phi$ $=0^{\circ}$ ) creates a net positive charge on one corner and a negative on the adjacent corner [the left side of the patch in Fig. 5 (b)] but on the right-hand side the electric field direction. Therefore, the charge distribution is reversed due to phase delay across the patch if the wave is incident off normal. This reversal creates the requisite charge distribution and permits coupling.

A $p$-polarized wave incident off normal and at $\phi=45^{\circ}$ [Fig. 5(c)] has components of its electric field (shown by dashed lines) parallel to all sides of the patch. Again, the phase reversal across the patch drives charges of like sign to diagonally opposite corners, making coupling to the $\{1,1\}$ mode possible. As for $\phi=0^{\circ}$, the requirement for a phase change across the patch prevents coupling at normal incidence. On first inspection, an off-normal $s$-polarized wave at $\phi=45^{\circ}$ has components of an electric field parallel to the edges of the patch and should therefore couple to the $\{1,1\}$ mode. However, the field direction shown in Fig. 5(d) drives charges of opposite rather than like sign toward diagonally opposite corners, and hence the requisite charge distribution is not created. 

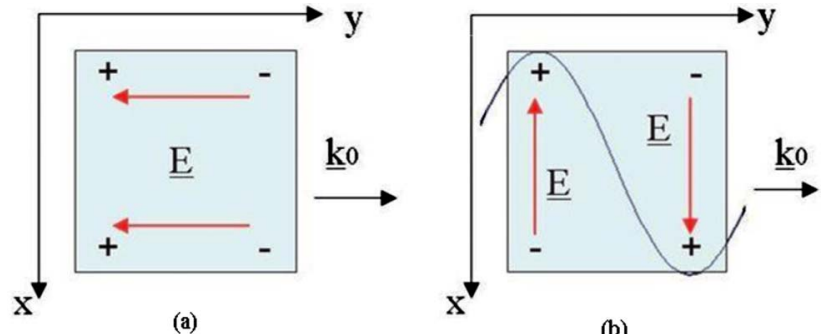

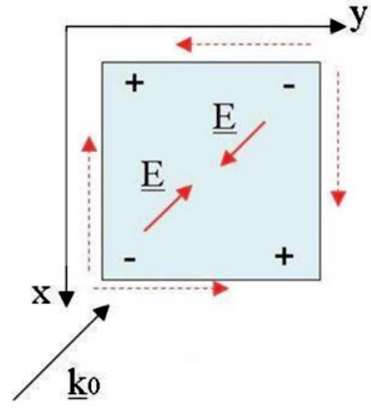

(c)

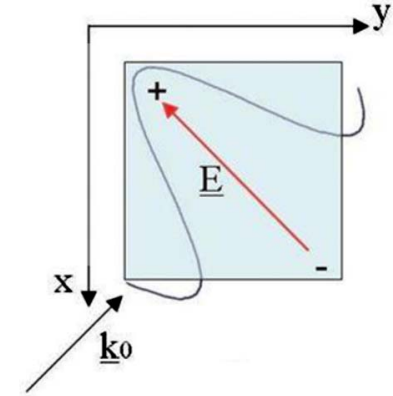

(d)
FIG. 5. (Color online) Bigrating sample. (a) Incident wavevector and electric vectors on the lower surface of a metal patch and the resulting charge distribution for $\phi=0^{\circ}, p$-polarization, (b) $\phi=0^{\circ}, s$-polarization, (c) $\phi=45^{\circ}$, $p$-polarization, and (d) $\phi=45^{\circ}, s$-polarization.

The second order $(2,0)$ mode, which is visible between 14 and $15 \mathrm{GHz}$ in Fig. 4(a), shows significant curvature due to the change in momentum available to the mode as the grating is rotated, as is the case for the monograting [see Eq. (2)]. However, on the rotation of the bigrating away from $\phi= \pm 90^{\circ}$, coupling to the $(0,2)$ mode associated with the second orthogonal set of slits becomes possible. At $\phi=45^{\circ}$ the two modes become degenerate and the two curved bands intersect; upon reaching $\phi= \pm 90^{\circ}$ the mode is wholly the $(0$, 2) mode.

Plots of the electric field vector on the lower metal layer for the $\{2,0\}$ modes are shown in Figs. 6(a)-6(c). At $0^{\circ}$ azimuth the $(2,0)$ mode [Fig. 6(a)] has electric field antinodes at the edges of the patch parallel to the $x$-axis and also across the center of the patch. At $\phi= \pm 90^{\circ}$ the $(0,2)$ mode has antinodes along the patch edges parallel to the $y$-axis and in the center of the patch [Fig. 6(b)]. The degeneracy of the $(2,0)$ and $(0,2)$ modes at $\phi= \pm 45^{\circ}$ means that the field pattern at this azimuth is simply the superposition of the fields from the $(2,0)$ and $(0,2)$ modes [Fig. 6(c)]. The curved bands between 16.5 and $17.5 \mathrm{GHz}$ are also absent from the monograting due to the excitation of the $\{2,1\}$ modes; a plot of the electric field vector for a $\{2,1\}$ mode is shown in Fig. 6(d).

A grayscale plot of $R_{s s}$ at a polar angle of $57^{\circ}$ is shown in Fig. 4(b). As for $p$-polarization, the fundamental is nondispersive and the $\{1,1\}$ modes appear at $\approx 11 \mathrm{GHz}$. In this case, the mode cannot be coupled to at $45^{\circ}$ azimuth, as explained above. The second order mode appears between 14 and $15 \mathrm{GHz}$ and behaves in a very similar manner to the second order of the monograting, the main difference being the degeneracy of the $(2,0)$ and $(0,2)$ modes at $45^{\circ}$ azimuth. The $\{2,1\}$ modes are visible between 16.5 and $17.5 \mathrm{GHz}$.

Finally we track the resonant frequency of each of the modes as a function of the incident angle to give their dispersion curves. This has been done for azimuthal angles of $0^{\circ}$ and $45^{\circ}$ for both $p$-polarized and $s$-polarized radiations; the dispersion curves are shown in Figs. 7(a) and 7(b), respectively. It can be seen that each mode is relatively flat

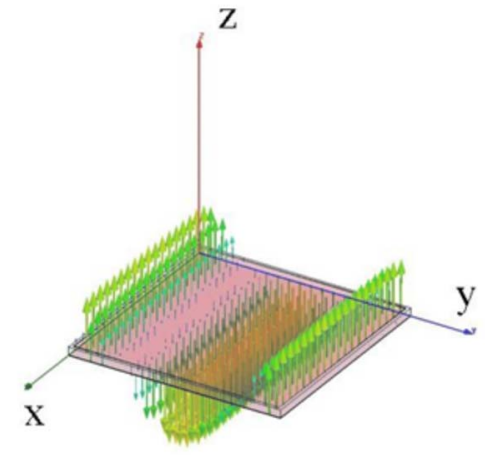

(a)

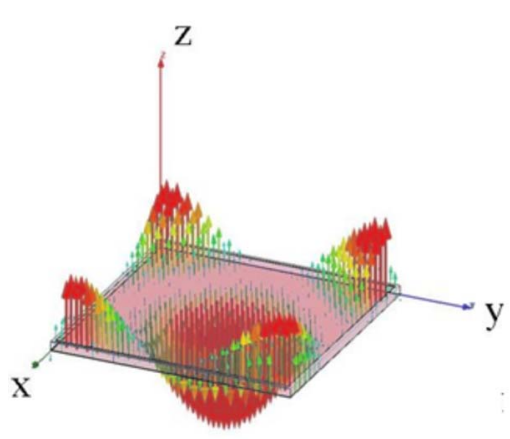

(c)

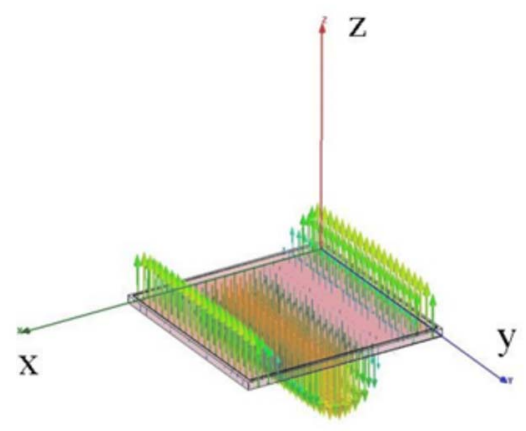

(b)

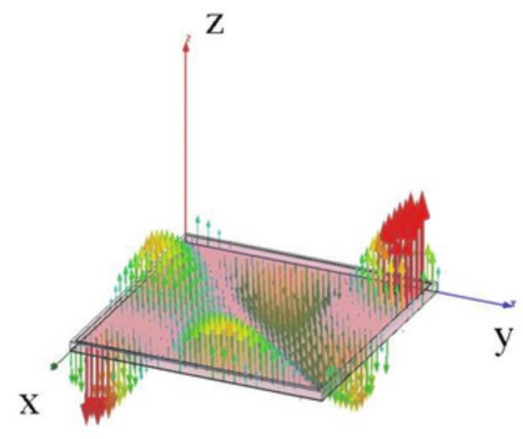

(d)
FIG. 6. (Color online) Distribution of the electric field on the upper surface of the lower metal layer plotted at a phase corresponding to maximum field. (a) The $(2,0)$ mode at $\phi=90^{\circ}$ and $13.9 \mathrm{GHz}$. (b) The $(0,2)$ mode at $\phi=0^{\circ}$ and $13.9 \mathrm{GHz}$. (c) The degenerate $(2,0)$ and $(0,2)$ modes at $\phi=45^{\circ}, 14.55 \mathrm{GHz}$. (d) The $(2,1)$ mode at $\phi=45^{\circ}, 16.6 \mathrm{GHz}$. 


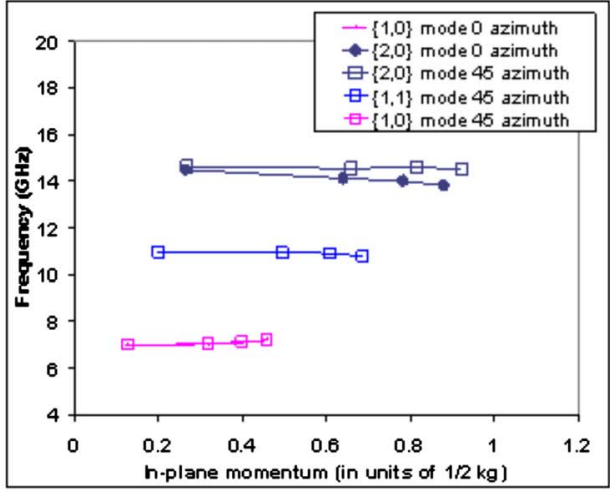

(a)

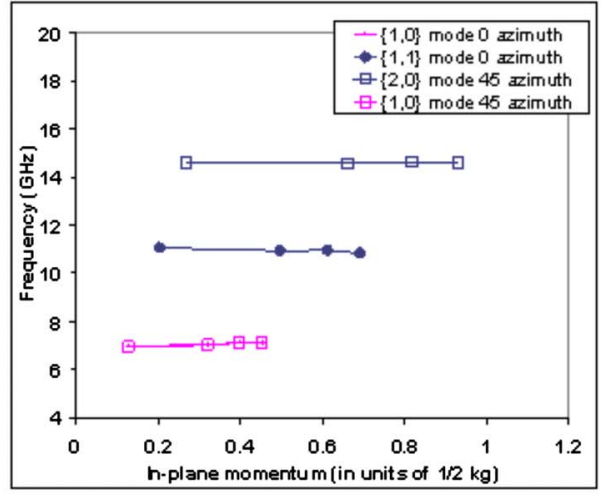

(b)
FIG. 7. (Color online) Dispersion plots determined from the frequency of the modes supported by the bigrating sample at $\phi=0^{\circ}$ and $45^{\circ}$ with (a) $p$-polarized and (b) $s$-polarization incident radiation. banded. However, the resonant frequency of the $\{1,0\}$ mode increases very gradually with increasing angle. By contrast, the frequency of the $\{1,1\}$ modes decreases slightly. This occurs for both polarization states at $0^{\circ}$ and $45^{\circ}$ azimuths. The $\{2,0\}$ modes do not vary in frequency at $45^{\circ}$ azimuth for either polarization but do decrease in frequency with increasing polar angle at $0^{\circ}$ azimuth for $p$-polarization.

\section{CONCLUSIONS}

Experimental measurements have demonstrated that the ultrathin attenuating structure studied previously, which selectively absorbed one polarization of incident radiation, is readily improved by patterning in two dimensions to absorb strongly any polarization. In addition, some of the resonant modes exhibit a high degree of azimuthal and polar-angle independent electromagnetic responses, thus enabling the absorption of both TE and TM polarized radiations over a wide range of angles. This characteristic can clearly be exploited to create lightweight, thin, low-cost absorbers as well as other structures, which can be used, for example, to minimize stray reflections from a radio frequency identification system and enable deployment in a cluttered, high-metal environment such as a warehouse. It may also be possible to exploit the compact nature of this attenuating material to improve electromagnetic compatibility by using to line the inside of cases housing electronic equipment.

It has also been demonstrated that the behavior of these ultrathin absorbing structures can be fully predicted, even at off-normal incidence, using finite element modeling. This approach has been used to examine the electromagnetic character of the modes and has revealed that in contrast to the previously studied structure, the two-dimensional array supports coupled modes having both $x$ - and $y$-components. The two-dimensional array also supports higher-order modes that cannot be excited by the one-dimensional structure, which results in a series of discrete absorption bands. The frequencies and azimuthal angles at which these higher-order modes can be coupled to could be used as the basis for a compact radio frequency sensing, orientation, or identification system.

\section{ACKNOWLEDGMENTS}

The authors are grateful to the EPSRC-GB, QinetiQ, and Omni-ID for financial support.

${ }^{1}$ E. F. Knott, J. F. Schaeffer, and M. T. Tuley, Radar Cross Section, 2nd ed. (Artech House, Norwood, MA, 1993), Chap. 8.

${ }^{2}$ W. W. Salisbury, U.S. Patent No. 2,599,944 (10 June 1952).

${ }^{3}$ A. P. Hibbins, J. R. Sambles, C. R. Lawrence, and J. R. Brown, Phys. Rev. Lett. 92, 143904 (2004).

${ }^{4}$ A. P. Hibbins, W. A. Murray, J. Tyler, S. Wedge, W. L. Barnes, and J. R. Sambles, Phys. Rev. B 74, 073408 (2006).

${ }^{5}$ Ying Zhang, J. von Hagen, M. Younis, C. Fischer, and W. Wiesbeck, IEEE Trans. Antennas Propag. 51, 2704 (2003).

${ }^{6}$ D. F. Sievenpiper, J. H. Schaffner, H. J. Song, R. Y. Loo, and G. Tangonan, IEEE Trans. Antennas Propag. 51, 2713 (2003).

${ }^{7}$ D. Sievenpiper, L. J. Zhang, R. F. J. Broas, N. G. Alexpolous, and E. Yablonovitch, IEEE Trans. Microwave Theory Tech. 47, 2059 (1999).

${ }^{8}$ Q. Gao, D.-B. Yan, N.-C. Yan, and Y. Yin, Electron. Lett. 41, 936 (2005).

${ }^{9}$ A. Tennant and B. Chambers, IEEE Microw. Wirel. Compon. Lett. 14, 46 (2004).

${ }^{10}$ A. Tennant and B. Chambers, Smart Mater. Struct. 13, 122 (2004).

${ }^{11}$ High Frequency Structure Simulator, Ansoft Corporation, Pittsburgh, PA, USA.

${ }^{12}$ Eurotech Group plc., Exmouth, UK.

${ }^{13}$ I. S. Grant and W. R. Phillips, Electromagnetism, 2nd ed. (Wiley, Chichester, 1995), Chap. 12

${ }^{14}$ Y. Takakura, Phys. Rev. Lett. 86, 5601 (2001). 